\title{
ELEMENTOS FACILITADORES DO ENTENDIMENTO DAS INTERFACES ENTRE SISTEMAS NATURAIS E SOCIOECONÔMICOS
}

\author{
D. C. ANDRADE \\ Instituto de Economia da Universidade Federal de Uberlândia \\ caixetaandrade@ie.ufu.br
}

Artigo submetido em maio/2014 e aceito em março/2015

DOI: $10.15628 /$ holos.2015.2125

\section{RESUMO}

A natureza multidimensional dos desafios trazidos pela crescente perda do capital natural impõe a necessidade de estratégias inovadoras capazes de assegurar um fluxo contínuo e sustentável de prosperidade humana. Tais estratégias, porém, apenas terão êxito se partirem de uma de base sólida de conhecimento sobre os determinantes do bem-estar humano e suas interfaces com o patrimônio natural da humanidade. Mas, diante da complexidade e crescente interconectividade de fenômenos como as mudanças climáticas, a crescente perda de biodiversidade e a pobreza persistente, quais são as estratégias disponíveis para facilitar o entendimento das interações dinâmicas entre sistemas naturais e socioeconômicos? Este artigo postula que, de um lado, a abordagem de serviços ecossistêmicos pode trazer importantes contribuições de natureza analítica e conceitual para a construção de uma agenda de pesquisa interdisciplinar na qual seja explicitamente reconhecida a interdependência entre capital natural e prosperidade humana. Isto porque os serviços ecossistêmicos são a interface básica entre capital natural e bem-estar humano. De outro lado, argumentase ainda que as interações mútuas entre sociedade e natureza podem ser melhor compreendidas por meio do uso de ferramentas operacionais como a modelagem econômico-ecológica.

PALAVRAS-CHAVE: Capital natural. Serviços ecossistêmicos. Bem-estar humano. Modelagem econômicoecológica. Pesquisa interdisciplinar.

\section{ENABLING THE COMPREHENSION OF THE INTERFACES BETWEEN NATURAL AND SOCIOECONOMIC SYSTEMS}

\begin{abstract}
The multidimensional nature of challenges delivered by the increasing loss of natural capital imposes the need for innovative strategies to ensure a continuous and sustainable flow of human prosperity. Such strategies, however, will only be successful if they are based on a solid knowledge backbone on the determinants of human well-being and their interfaces with humanity's natural wealth. Due to the increasing complexity and interconnectedness of phenomena such as climate change, biodiversity loss and persistent poverty, what are the strategies available to facilitate the understanding of the dynamics interactions between
\end{abstract}

natural and socioeconomic systems? On one hand, this paper advocates that the approach of ecosystem services can provide important analytical and conceptual contributions to build an interdisciplinary research agenda in which the interdependence between natural capital and human prosperity is explicitly recognized. This is because ecosystem services are known as the basic interface between natural capital and human welfare. On the other hand, it is argued that the mutual interactions between society and nature can be better understood by adopting some operational tools such as the ecological-economic modeling.

KEYWORDS: Natural capital. Ecosystem services. Human well-being. Ecological-economic modeling. Interdisciplinary research. 


\section{INTRODUÇÃO}

Em um contexto de crescente vulnerabilidade ambiental, social e econômica, são cada vez mais necessárias soluções capazes de assegurar incrementos equitativos e sustentáveis do bemestar humano. Mas, como desenhar e implementar estratégias de intervenção eficazes e compatíveis com o nível de complexidade dos fenômenos reais? De maneira específica, como potencializar o conhecimento sobre as interações e interfaces entre sistemas naturais e socioeconômicos?

As respostas aos questionamentos acima certamente devem partir de um esforço interdisciplinar de pesquisa. A partir desse reconhecimento, este trabalho objetiva discutir elementos facilitadores que podem contribuir para tornar o diálogo interdisciplinar mais eficaz, permitindo um entendimento holístico da interdependência mútua entre sistemas naturais e socioeconômicos.

A hipótese trabalhada é de que, de um lado, a abordagem de serviços ecossistêmicos (ASE) pode trazer importantes contribuições analíticas e conceituais para a construção de uma agenda de pesquisa interdisciplinar na qual seja explicitamente reconhecida a interdependência entre capital natural e prosperidade humana. De outro lado, argumenta-se que as interações mútuas entre sociedade e natureza podem ser melhor compreendidas por meio do uso de ferramentas operacionais como a modelagem econômico-ecológica (MEE).

Todos os argumentos desenvolvidos partem da premissa básica de que os serviços ecossistêmicos são a interface básica entre capital natural e bem-estar humano. Logo, a possibilidade de prosperidade contínua da espécie humana está diretamente ligada à capacidade de preservação do capital natural ${ }^{1}$.

O arcabouço teórico que sustenta os argumentos deste trabalho são provenientes da chamada economia ecológica (ÖZKAYNAK et al., 2012), a qual reconhece o funcionamento termodinâmico do sistema econômico e sua dependência estrita em relação aos sistemas naturais. Ademais, a economia ecológica possui uma visão pré-analítica de que o sistema econômico é um subsistema aberto da ecosfera e, portanto, tem sua expansão física restringida pelos limites oferecidos pela resiliência dos ecossistemas. É neste sentido que é necessária a adoção de ferramentas teóricas e metodológicas que facilitem a compreensão das inter-relações complexas entres sistemas naturais e socioeconômicos, tendo como princípio básico uma perspectiva econômico-ecológica.

O trabalho está dividido em três seções. A primeira revisa brevemente as principais interações entre ecossistemas e o sistema econômico. A segunda seção apresenta os conceitos de serviços ecossistêmicos e suas contribuições para o bem-estar humano. Especial ênfase é dada ao papel crucial dos serviços ecossistêmicos para a obtenção de objetivos de desenvolvimento socioeconômico. A terceira seção argumenta que, no âmbito analítico e conceitual, a ASE pode trazer importantes contribuições para análises interdisciplinares das

\footnotetext{
${ }^{1}$ Neste trabalho, o termo capital natural designa o conjunto de ecossistemas existentes. Por conseguinte, o capital natural representa o patrimônio natural da humanidade ou, ainda, o portfólio disponível de ativos naturais tangíveis e intangíveis. De modo geral, indica que a fonte original de todas as atividades humanas é efetivamente a riqueza natural da ecosfera.
} 
relações entre sociedade e meio ambiente. Adicionalmente, discute-se ainda que, do ponto de vista metodológico, o uso da ferramenta operacional de MEE facilita a compreensão das interfaces entre ecossistemas, seus serviços e bem-estar humano.

\section{SISTEMAS NATURAIS E SOCIOECONÔMICOS: INTERFACES BÁSICAS}

Os sistemas naturais, conhecidos genericamente por ecossistemas, englobam as complexas, dinâmicas e contínuas interações entre seres vivos e não vivos em seus ambientes físicos e biológicos em que o homem é parte integral (MEA, 2003). São sistemas adaptativos complexos, nos quais propriedades sistêmicas macroscópicas como estrutura, relação produtividade-diversidade e padrões de fluxos de nutrientes emergem de interações entre os componentes, sendo comum a existência de efeitos de retroalimentação ("feeedback") (LEVIN, 1998), numa combinação de efeitos negativos e positivos responsáveis por um equilíbrio dinâmico e evolutivo. Eles incluem não apenas as interações entre os organismos, mas entre a totalidade complexa dos fatores físicos que formam o que é conhecido como ambiente (TANSLEY, 1935).

Enquanto sistemas complexos, os ecossistemas apresentam várias características (ou propriedades), como variabilidade, resiliência, sensibilidade, persistência, confiabilidade, etc. Dentre elas, as propriedades de variabilidade e resiliência apresentam importância crucial para uma análise integrada das interconexões entre ecossistemas, sistemas socioeconômicos e bemestar humano.

Os ecossistemas são profundamente modificados pela ação humana. O sistema econômico - veículo principal da ação humana - interage com o meio ambiente, extraindo recursos naturais e energéticos (componentes estruturais dos ecossistemas) de baixa entropia e devolvendo resíduos de alta entropia ${ }^{2}$. Além disso, altera consideravelmente o espaço em que atua em função de sua expansão ${ }^{3}$. Assim, o funcionamento do sistema econômico traz impactos quantitativos e qualitativos sobre os ecossistemas, sendo tais impactos função da escala (tamanho, dimensão) e do estilo dominante de crescimento econômico (modo pelo qual o sistema econômico se expande).

É possível apontar três perspectivas teóricas que buscam analisar os impactos ambientais provocados pela intervenção antrópica sobre os ecossistemas (YORK et al., 2003). A primeira delas, conhecida como abordagem da ecologia humana, aplica princípios ecológicos para compreender as sociedades humanas, afirmando que, embora a capacidade do homem no que diz respeito à organização, tecnologia e cultura o distancie das demais espécies, suas ações são sempre limitadas por condições ecológicas.

A segunda, neoclássica, aponta para os efeitos da modernização (tecnológica, econômica e institucional) sobre o grau de impacto que as atividades humanas podem ter sobre os ecossistemas, sugerindo que os problemas ambientais globais podem ser resolvidos por meio de

\footnotetext{
${ }^{2}$ Este fato atesta a natureza entrópica do funcionamento do sistema econômico (GEORGESCU-ROEGEN, 1971). Esta interpretação termodinâmica se afasta do mecanicismo característico da teoria econômica convencional (CHECHIN; VEIGA, 2010).

${ }^{3} \mathrm{Na}$ terminologia convencional, a expansão física do sistema econômico é conhecida como crescimento econômico, que é tradicionalmente medida pelo incremento no Produto Interno Bruto (PIB) das nações.
} 
modificações em instituições econômicas e sociopolíticas, sem necessariamente renunciar (ou reduzir) ao crescimento econômico, ao capitalismo e à globalização (YORK et al., 2003).

Este otimismo em relação à degradação ambiental está em contraste com a terceira perspectiva, da economia política da sustentabilidade, segundo a qual o conflito existente entre economia e ecossistemas apenas será resolvido a partir de reestruturação radical da sociedade e de imposição de limites à expansão contínua da produção (e consumo). Dentro desta perspectiva, a reversão dos processos de degradação dos ecossistemas e o alcance da chamada sustentabilidade forte só podem ser obtidos por meio de uma reestruturação dos valores culturais da sociedade. Esta mudança institucional poderia alterar o modus operandi das sociedades, substituindo o objetivo máximo de acumulação de riqueza material pela acumulação de riqueza espiritual propiciada por atividades sociais e culturais (ROMEIRO, 2000).

A transição para uma trajetória de evolução sustentável deve partir do reconhecimento de que os sistemas naturais são, em última instância, a fonte primordial de bem-estar humano. Entretanto, pouco se tem avançado no entendimento sobre a dinâmica subjacente aos ecossistemas e suas interações com as variáveis socioeconômicas. É neste sentido, pois, que se reconhece a utilidade da ASE como instrumento de conexão entre as perspectivas das ciências naturais e sociais. Os conceitos pertinentes a este argumento são apresentados na próxima seção.

\section{SERVIÇOS ECOSSISTÊMICOS E BEM-ESTAR HUMANO}

É possível encontrar várias definições de serviços ecossistêmicos (WALLACE, 2007). Este trabalho explicitamente adota o conceito utilizado pela Avaliação Ecossistêmica do Milênio, segundo a qual "ecosystem services are the benefits people obtain from ecosystems" (MEA, 2005, p. 1) ${ }^{4}$. Dentre eles, pode-se citar a provisão de alimentos, a regulação climática, a formação do solo, etc. (DAILY, 1997; COSTANZA et al., 1997; DE GROOT et al., 2002; MEA, 2003). "[Ecosystems] Services are therefore actually conceptualizations ('labels') of the 'useful things' ecosystem 'do' for people, directly and indirectly" (BRAAT; DE GROOT, 2012, p. 6).

Um dos principais componentes da estrutura dos ecossistemas é a chamada biodiversidade, que pode ser definida como a variabilidade entre os organismos vivos e entre os ecossistemas terrestres, marinhos e outros ecossistemas aquáticos, além de todos os processos ecológicos dos quais tais organismos fazem parte (UNEP, 2004). A perda da biodiversidade representa a maior ameaça aos ecossistemas e à sua capacidade de sustentar processos ecológicos básicos que suportam a vida no planeta (NAEEM et al., 1999).

Alguns autores afirmam que a popularização do termo serviços ecossistêmicos se deveu ao objetivo explícito de enfatizar os efeitos negativos sobre o bem-estar humano decorrentes da perda crescente de biodiversidade (BRAAT; DE GROOT, 2012). Logo, são indissociáveis os estudos sobre os serviços ecossistêmicos e biodiversidade.

Os processos (funções) e serviços ecossistêmicos nem sempre apresentam uma relação biunívoca, sendo que um único serviço ecossistêmico pode ser o produto de duas ou mais

\footnotetext{
${ }^{4} \mathrm{O}$ conceito empregado se refere aos bens ecossistêmicos (recursos tangíveis) e serviços propriamente ditos (benefícios intangíveis), originados pelas complexas interações entre os componentes de ecossistemas naturais e cultivados.
} 
funções, ou uma única função pode gerar mais que um serviço ecossistêmico (COSTANZA et al., 1997; DE GROOT et al., 2002). A natureza interdependente das funções ecossistêmicas (processos ecológicos) faz com que a análise de seus serviços requeira a compreensão das interconexões existentes entre os seus componentes, resguardando a capacidade dinâmica dos ecossistemas em gerar seus serviços (LIMBURG; FOLKE, 1999).

Como resultado da degradação dos ecossistemas, a ameaça de alterações drásticas nos fluxos de serviços ecossistêmicos tem crescentemente preocupado a comunidade acadêmica e os tomadores de decisão. Parafraseando Hardin (1968), Lant et al. (2008) utilizam a expressão "tragédia dos serviços ecossistêmicos" para se referirem ao declínio da sua oferta, principalmente considerando os serviços de regulação, de suporte e culturais ${ }^{5}$. Para estes autores, a degradação dos fluxos de serviços ecossistêmicos faz parte de uma armadilha social em que as falhas nas leis de propriedade comunal e os incentivos econômicos que abrangem apenas bens e serviços transacionados nos mercados são responsáveis pela destruição dos serviços de suporte à vida, oferecidos "gratuitamente" pelos ecossistemas.

Em um cenário de contínua degradação dos ecossistemas, o alcance do desenvolvimento sustentável requer um melhor entendimento da medida da dependência humana com relação aos serviços ecossistêmicos e, por conseguinte, da vulnerabilidade do bem-estar humano em relação às mudanças nos ecossistemas (EFTEC, 2005).

Os impactos de mudanças nos fluxos de serviços ecossistêmicos sobre os constituintes do bem-estar são complexos e envolvem relações de causação que se reforçam mutuamente, devido principalmente à interdependência dos processos de geração dos serviços ecossistêmicos e entre as próprias dimensões do bem-estar. As mudanças nos serviços ecossistêmicos de provisão, por exemplo, afetam todos os constituintes do bem-estar material dos indivíduos.

A degradação dos ecossistemas e as alterações nos fluxos de serviços ecossistêmicos podem também representar um sério entrave ao desenvolvimento socioeconômico. Os chamados "Objetivos de Desenvolvimento do Milênio" (SUKHDEV, 2008; MEA, 2005), estabelecidos em setembro de 2000 pelo Projeto do Milênio das Nações Unidas (UN Millennium Project, 2005), têm como premissa básica aumentar o bem-estar humano por meio da redução da pobreza, do combate à fome e à mortalidade materna e infantil, do acesso universal à educação, do controle de doenças, do fim das desigualdades entre homens e mulheres, do desenvolvimento sustentável e da construção de parcerias globais para o desenvolvimento.

De acordo com Andrade e Romeiro (2009), o papel dos serviços ecossistêmicos é crucial no alcance de tais objetivos, o que pode ser evidenciado pela constatação de que as regiões com maiores dificuldades em atingir as metas são aquelas onde suas populações apresentam uma maior dependência em relação aos ecossistemas e aos seus serviços (MEA, 2005). É evidente, pois, que qualquer ação que vise aumentar a qualidade de vida das populações e acelerar o processo de desenvolvimento deve reconhecer explicitamente a importância dos serviços prestados pelos ecossistemas para as condições de vida humana.

Conclui-se, portanto, que os serviços ecossistêmicos são a interface básica entre capital natural e prosperidade humana. Em decorrência disso, pode-se afirmar que estratégias bem-

\footnotetext{
${ }^{5}$ Segundo a classificação da Avaliação Ecossistêmica do Milênio, os serviços ecossistêmicos são divididos em 4 categorias: provisão (abastecimento), regulação, culturais e suporte. Para maiores detalhes, consultar MEA (2003).
} 
sucedidas para assegurar aumentos contínuos e sustentáveis da prosperidade humana dependem da capacidade de reversão da trajetória de degradação do capital natural. Por sua vez, políticas de preservação dos ecossistemas devem partir de uma base sólida de conhecimento sobre as interfaces entre sistemas naturais e socioeconômicos, o que muitas vezes não está ao alcance de pesquisadores, dificultando sobremaneira o diálogo interdisciplinar.

Diante dessa problemática, como tornar mais palatável e acessível o entendimento integrado da relação entre sistemas naturais e sociais? A próxima seção apresenta argumentos iniciais para responder a este questionamento.

\section{ASE E MEE COMO ELEMENTOS FACILITADORES DA PESQUISA INTERDISCIPLI- NAR}

Esta seção apresenta a principal proposição deste artigo ao argumentar que a ASE e a MEE são elementos que podem contribuir para o enriquecimento das pesquisas interdisciplinares com foco nas interfaces entre sociedade e natureza. De um lado, a ASE pode ser vista com uma ferramenta analítica e conceitual para a compreensão da dependência recíproca entre ecossistemas e bem-estar humano; de outro, a MEE é uma opção metodológica útil para operacionalizar o conhecimento de tais interações entre sistemas naturais e socioeconômicos.

Em se tratando da $A S E$, sua maior contribuição está no fato de que ela redefine o relacionamento entre natureza e o resto da humanidade (CONTANZA, 2011). O seu alicerce básico está na tentativa de se estabelecer uma conexão entre ciências naturais e sociais. Em decorrência disso, serviços ecossistêmicos são vistos como um bridging concept, cuja função precípua é reforçar e consolidar o conhecimento sobre a dependência humana em relação ao capital natural (BRAAT; DE GROOT, 2012). Para tanto, a ASE pressupõe a integração científica de perspectivas ecológicas, socioeconômicas e institucionais como requisito para análises plurais sobre as interações e feedbacks entre ambientes naturais e construídos.

Do ponto de vista do debate sobre desenvolvimento sustentável, a ASE é elemento importante como contraponto aos argumentos de que o capital natural pode ser substituído pelo capital produzido pelo homem. Isto porque o reconhecimento dos serviços ecossistêmicos reforça a existência de benefícios intangíveis, cuja substituição pode ser altamente custosa ou mesmo impossível (RESENDE et al., 2014). No lugar da concepção de substituibilidade, conhecida como sustentabilidade fraca, a incorporação do conceito de serviços ecossistêmicos fortalece a interpretação de complementaridade entre os diversos tipos de capital, além do que corrobora o argumento de que a depleção do meio ambiente conduz a perdas quantitativas e qualitativas. Neste sentido, a ASE contribui para tornar mais robusta a perspectiva de sustentabilidade forte.

Com base nos argumentos de Andrade et al. (2012), pode-se dizer que a ASE é importante porque amplia a problemática da escassez do capital natural enquanto tema relevante para a discussão sobre a sustentabilidade das atividades econômicas. Para estes autores, desconsiderar o papel do capital natural enquanto provedor de serviços fundamentais ao bem-estar humano significa não reconhecer a interdependência entre estrutura e funções do capital natural e sua resiliência. Em sendo assim, a incorporação da ideia de que o capital natural é um fundo de serviços essenciais à manutenção das condições mínimas de vida (em essência, a ASE) propicia 
um enfrentamento realista e não sofista dos desafios colocados por um estudo desvelado das relações entre sistemas naturais e socioeconômicos.

Ainda no âmbito da ideia de sustentabilidade, o tratamento do capital natural como fundo de serviços e a emergência da ASE resultou em uma alteração na ênfase dada às origens dos problemas ambientais. Até a década de 1970, a tônica das preocupações com a sustentabilidade estava na dimensão de fornecedor de recursos naturais e energéticos (bens tangíveis) do meio ambiente. Entretanto, a emergência do conceito de serviços ecossistêmicos fez com que maior realce fosse destinado à oferta de benefícios intangíveis de suporte às atividades socioeconômicas, especialmente aos serviços de absorção dos resíduos gerados pela produção de bens e serviços. Nesta perspectiva, fenômenos indesejáveis como as mudanças climáticas, por exemplo, são o resultado da degradação contínua de serviços básicos oferecidos pelos sistemas naturais ${ }^{6}$. A ASE oferece, portanto, novas formas de enquadramento dos problemas ambientais de modo a facilitar o seu diagnóstico e enfrentamento.

No que diz respeito à MEE, esta ferramenta metodológica permite potencializar e operacionalizar a geração de conhecimentos sobre as interfaces entre os sistemas naturais e socioeconômicos. A descrição dos sistemas naturais (ecossistemas) tem se mostrado um campo fértil de pesquisas (GOMES; VARRIALE, 2004), e uma característica marcante desse desafio é a transversalidade com que deve ser tratada a sua representação, visto que a complexidade dos fenômenos ecológicos exige a junção de várias disciplinas.

Um fator distintivo das representações dos ecossistemas é a não linearidade das equações de movimento que descrevem as interações e os processos ecológicos complexos. Somado a isso, tem-se também a aleatoriedade dos fenômenos (GOMES; VARRIALE, 2004), o que torna ainda mais instigante a tarefa de representação ecossistêmica.

O primeiro passo para a representação dos ecossistemas é a compreensão mínima de como estes se organizam internamente e quais são suas tendências gerais de evolução e sobrevivência. A partir dos seus princípios gerais de funcionamento, pode-se partir para a representação dos ecossistemas, que tem sido feita por meio da construção de modelos ecossistêmicos (ou ecológicos). De maneira mais simples, um modelo pode ser considerado como uma representação simplificada da realidade (VOINOV, 2008), na qual são descritas as interações que se consideram mais importantes para representar o funcionamento de um ecossistema. Com efeito, o ponto de partida fundamental para a modelagem ecossistêmica é a seleção dos seus componentes e processos ecologicamente mais relevantes sem prejuízo para a compreensão básica de sua estrutura e dinâmica (GOMES;VARRIALE, 2004).

Um modelo pode ser descrito como uma representação proposital de um sistema, que consiste em elementos estruturais e suas relações internas, além de inter-relações destes com os ambientes subjacentes. As especificações dos elementos estruturais e dos relacionamentos internos e externos determinam em que medida um modelo pode ser considerado integrado e interdisciplinar.

\footnotetext{
${ }^{6}$ Neste caso, as mudanças climáticas em última instância são a consequência da perda contínua da capacidade de regulação climática, cuja causa primária é a emissão de gases de efeito estufa além da capacidade natural de sua absorção pela atmosfera.
} 
A construção dos modelos necessariamente deve usar uma abordagem sistêmica ou uma abordagem que se pode chamar de system thinking (VON BERTALANFFY, 1975). Para Voinov (2008. p. 6), "a system is a combination of parts that interact and produce some new quality in their interaction". Tal definição pressupõe a existência de três características básicas dos sistemas: i) suas partes ou elementos; ii) as interações que ocorrem entre seus elementos (majoritariamente regidas por relações não lineares); iii) o resultado dessas interações.

Independente de como são apresentados, a necessidade do uso dos modelos se deve à incapacidade cognitiva de se entender de imediato e simultaneamente todas as interações e variáveis que afetam um sistema complexo. Sendo assim, é necessário que se adote um mecanismo de compreensão processual, de onde se parte de estruturas analíticas simplificadas para estruturas mais completas. A função dos modelos é justamente auxiliar o cientista na organização e construção dessas estruturas analíticas mais simples que servem, ao mesmo tempo, como instrumento para a análise da dinâmica básica de um sistema e suporte para a compreensão mais holística de uma determinada realidade.

O uso de modelos para a compreensão mínima da dinâmica dos sistemas (ou ecossistemas) não é exclusivo das ciências naturais. As ciências sociais, entre elas as ciências econômicas, têm sido caracterizadas pela utilização intensiva de modelos econômicos como parte de um processo de crescente formalismo. São usados como auxílio para o esclarecimento de problemas econômicos, cuja natureza é essencialmente dialética.

Independente dos aspectos normativos sobre a utilização da modelagem econômica, o fato é que esta prática tem sido bastante disseminada. No entanto, seguindo a tradição neoclássica, tais modelos não incorporam a dimensão ecológica, como que numa espécie de "fantasia", na qual os fenômenos econômicos não têm relação com o meio ambiente e este não representa nenhum tipo de obstáculo à obtenção dos resultados econômicos.

Assim como os modelos econômicos, modelos ecossistêmicos frequentemente não trazem considerações sobre os impactos que atividades econômicas têm sobre o meio ambiente. Essa tendência de isolacionismo em ambos os casos pode limitar os resultados dos modelos, tornando-os inadequados para tratar simultaneamente as dimensões socioeconômica e ecológica dos fenômenos reais. Este fato representa um importante entrave para o êxito de pesquisas interdisciplinares que têm como foco o mapeamento e a compreensão das interações entre sociedade e ambiente.

A compreensão das ameaças ao fluxo sustentável de bem-estar humano proveniente dos serviços ecossistêmicos requer a integração de modelos econômicos e ecológicos. Tal integração exige o cumprimento de três requisitos necessários: i) profundo conhecimento das disciplinas envolvidas (no caso, ciências naturais e sociais); ii) identificação e estruturação adequada do problema a ser investigado, e; iii) entendimento mútuo entre os pesquisadores (cientistas naturais e sociais) sobre as escalas e os propósitos da ferramenta da MEE.

Segundo Costanza e Ruth (1998), os propósitos da modelagem dos sistemas econômicos e ecológicos podem variar entre o desenvolvimento de simples modelos conceituais, que fornecem um entendimento geral do comportamento do sistema modelado, a aplicações realistas, cujo objetivo é avaliar diferentes propostas de política. Os três critérios necessários para avaliar a eficiência dos modelos são suas características de realismo (simulação de um sistema de uma maneira qualitativamente realística), precisão (simulação de um sistema de uma maneira 
quantitativamente precisa) e generalidade (representação de um amplo intervalo de comportamentos sistêmicos com o mesmo modelo). Nenhum modelo poderá maximizar simultaneamente as três características, e a escolha do atributo a ser realçado dependerá dos propósitos fundamentais para os quais o modelo está sendo construído.

Particularmente, modelos econômico-ecológicos são essenciais para o planejamento do uso da terra. Nestes casos, deve-se levar em consideração os trade-offs envolvidos entre rendimentos econômicos e provisão de serviços ecossistêmicos. A escolha de um determinado uso da terra, por exemplo, pode maximizar os retornos econômicos no curto prazo, mas podem degradar um serviço ecossistêmico cuja escassez futura poderá comprometer a própria sustentabilidade do uso da terra escolhido. Por outro lado, estratégias de conservação do capital natural desconectadas de informações econômicas podem não encontrar aderência por parte dos principais agentes da conservação (stakeholders).

Outro exemplo em que a integração de modelos ecológicos e ecossistêmicos é desejável está na elaboração de zoneamentos ecológicos em áreas que apresentam um alto custo de oportunidade da terra. Uma estratégia de conservação para estas regiões deve levar em conta não apenas as prioridades de conservação da diversidade biológica, baseadas, principalmente, em atributos de relevância ecossistêmica, mas também incorporar dados econômicos, uma vez que o sucesso das políticas de conservação está diretamente relacionado a um adequado equilíbrio entre as variáveis ecológicas e econômicas.

Outra contribuição da MEE é a possibilidade de elaboração de cenários, considerados essenciais para a formulação de políticas e para a apreciação de resultados econômicos e ecológicos em função de vários tipos de estratégias adotadas. Uma vez apresentados os resultados dos cenários simulados, experts e sociedade civil podem conjuntamente decidir sobre aquela que melhor se adequa a critérios ecológicos, econômicos e sociais.

Apesar de suas vantagens, a disseminação da prática da MEE requer a superação de alguns desafios. Em primeiro lugar, estão aqueles inerentes à própria resistência de pesquisadores em adotar posturas interdisciplinares. Em segundo lugar, além do diálogo entre ciências naturais e sociais, a elaboração de modelos econômico-ecológicos exige que os profissionais dessas áreas cheguem a um consenso sobre as várias abordagens adotadas, sobre os procedimentos básicos de especificação dos modelos e escolha das escalas espaciais e temporais adequadas.

Um dos maiores obstáculos à construção de uma agenda de pesquisa efetivamente interdisciplinar está na tradição de divisão clássica das disciplinas, que dificulta a integração de várias perspectivas para o tratamento dos problemas ambientais e socioeconômicos. Mesmo que seja consenso que essa integração é necessária, a tarefa de amalgamar diferentes visões é altamente desafiadora em função das especificidades e peculiaridades de cada uma. Ademais, os fenômenos de degradação ambiental são causados por interações entre os sistemas ecológico e econômico, sendo ambos caracterizados por alta complexidade. Do lado ecológico, há questões sobre a irreversibilidade e resiliência dos ecossistemas; do lado econômico, as inovações, num sentido shumpeteriano, caracterizam este sistema como altamente dinâmico e imprevisível.

Nota-se, portanto, que a integração analítica das perspectivas naturais e sociais não é tarefa trivial. Todavia, este é um passo necessário para se potencializar as chances de sucesso de políticas que visam ao enfrentamento de problemáticas envolvendo a complexidade dos 
fenômenos sociais e naturais. Especificamente, políticas públicas com o objetivo de tornar a trajetória de desenvolvimento socioeconômico mais sustentável não devem prescindir de uma análise integrada das relações entre sistemas naturais e humanos.

Para enfrentar este desafio de integração de diferentes perspectivas, postula-se que a ASE e a MEE são, respectivamente, ferramentas conceitual e metodológica auxiliares para se contornar o reducionismo cartesiano, uma vez que permitem considerar simultaneamente os aspectos ecológicos, sociais e econômicos de determinada realidade. Em última instância, sua utilização facilita a compreensão da dinâmica ecossistêmica e os seus desdobramentos na esfera econômica. Da mesma forma, viabilizam o entendimento dos impactos das atividades antrópicas sobre o funcionamento dos processos ecológicos e sua capacidade de prover os serviços ecossistêmicos.

A consideração conjunta da ASE e MEE é compatível com a visão da economia ecológica, uma vez que tais abordagens incorporam implicitamente a visão pré-analítica diferenciadora de que o sistema econômico está contido na ecosfera e é por ela restringido. Ambas as abordagens deverão ser colocadas em prática a partir do princípio básico de que os sistemas econômicos não podem e não devem se expandir indefinidamente, já que existem limites biofísicos e econômicos para o incremento contínuo dos sistemas econômicos. Tanto a ASE quanto a MEE contribuem para o alcance do equilíbrio ecológico, social e econômico e são instrumentos úteis para o enfrentamento do aparente trade-off existente entre expansão econômica e preservação ambiental.

A figura 1 sistematiza as contribuições da ASE e MEE para a concepção de políticas públicas com a finalidade de prover meios para uma gestão e conservação prudente do capital natural. Em primeiro lugar, o reconhecimento da categoria de serviços ecossistêmicos (ASE) coloca explicitamente a natureza e a biodiversidade como elementos importantes para a evolução socioeconômica da humanidade. Em outras palavras, a incorporação da ASE faz com que os processos de tomada de decisão levem em consideração o papel da natureza na geração de bem-estar humano.

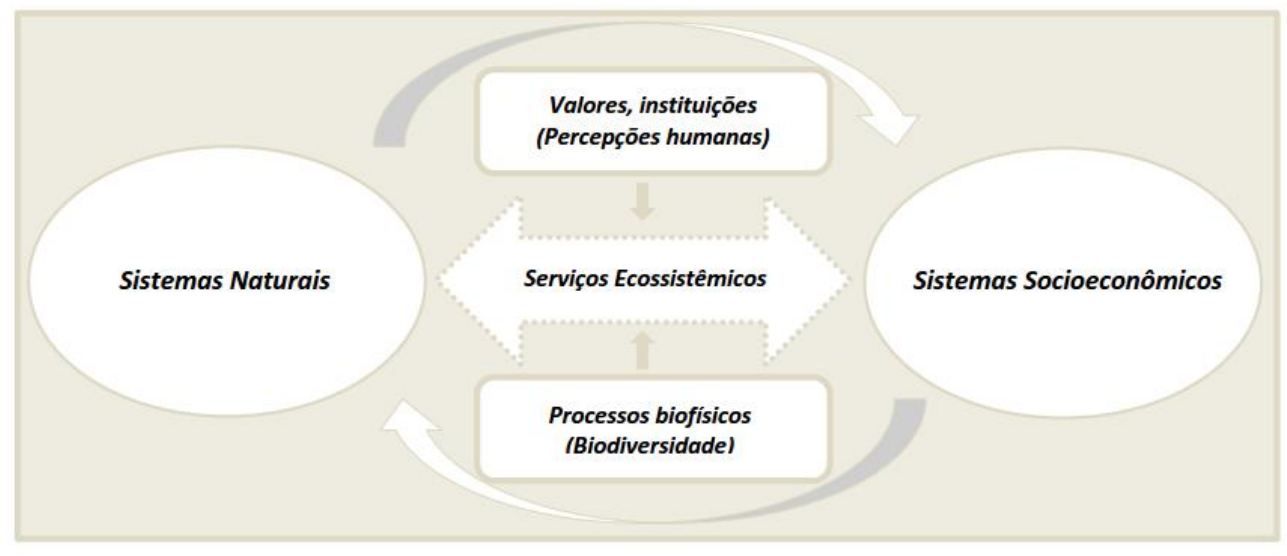

Políticas Públicas

(Gestão e Conservação)

(Desenvolvimento Sustentável)
Modelagem econômico-

ecológica

(Indicadores, Mapeamento) 
Figura 1 - ASE e MME: ferramentas conceitual e metodológica para o estudo das relações entre sistemas naturais e socioeconômicos

É preciso notar, contudo, que as instituições e a percepção humana são cruciais para que haja um apropriado julgamento da relevância dos serviços ecossistêmicos no suporte das atividades humanas. Este processo de atribuição de valores - sejam eles econômicos, ecológicos ou sociais - pode ser conhecido pela denominação genérica de valoração. De modo geral, o background cultural de uma determinada sociedade define sua capacidade de valorar e apreciar os serviços ecossistêmicos. Da mesma forma, sua evolução institucional e a dinâmica de valores socialmente acordados pode afetar positivamente a maneira pela qual a humanidade percebe os serviços ecossistêmicos.

Deve-se atentar para o caráter dinâmico, evolutivo e dialético das interações entre os dois sistemas representados na figura 1 . Isso demonstra a necessidade de conhecer os efeitos de retroalimentação, cuja intensidade pode ser alterada por meio das políticas públicas de gestão e conservação do capital natural. Tais políticas, por sua vez, devem ser constantemente redefinidas por meio de informações provenientes do mapeamento dos serviços ecossistêmicos, cuja viabilidade operacional depende de modelos econômico-ecológicos e da construção de um conjunto de indicadores de serviços ecossistêmicos.

Do ponto de vista da MEE enquanto ferramenta metodológica necessária, é preciso lidar com a natureza multiescalar dos serviços ecossistêmicos. Isso significa que todos os modelos e seus resultados devem ser interpretados de maneira a reconhecer as diferentes escalas temporais, espaciais e sociais. 0 mesmo se pode dizer com relação aos sistemas de indicadores, cuja função precípua é a de elucidar os aspectos negativos da perda de biodiversidade em termos da redução dos fluxos de serviços ecossistêmicos (MÜLLER; BURKHARD, 2012).

A afirmação de que os serviços ecossistêmicos operam em variadas escalas indica que os processos ecológicos que os geram podem ocorrer em níveis global, bioma, paisagem, ecossistema, frações de ecossistemas (plots) e em nível de plantas (HEIN et al., 2006). Ao mesmo tempo, os serviços ecossistêmicos afetam stakeholders em vários níveis institucionais (internacional, nacional, estadual/provincial, municipal, família e nível individual). Escalas ecológicas e níveis institucionais raramente coincidem, sendo que os diferentes stakeholders podem ter interesses distintos na gestão dos benefícios provenientes dos ecossistemas, baseados, principalmente, em dependências específicas com relação aos serviços gerados. Os problemas de escala (ecológica e institucional) reforçam a complexidade envolvida nos estudos de serviços ecossistêmicos, o que sugere que há uma necessidade premente de utilização de abordagens multiescalares e interdisciplinares (MARTíN-LÓPEZ et al., 2009).

Finalmente, todas as informações biofísicas devem estar conectadas com a evolução dos valores e instituições, cuja natureza é essencialmente social. A fim de se reduzir a lacuna existente entre reconhecimento dos serviços ecossistêmicos e sua efetivação na forma de políticas, é fundamental a eficiente sistematização do conhecimento gerado por análises interdisciplinares. Para tanto, estruturas de governança devem ser adaptadas de forma a permitir a utilização proveitosa do potencial representado pela ASE e MEE em pesquisas com foco na ideia ampla de sustentabilidade. 


\section{CONSIDERAÇÕES FINAIS}

É consensual a necessidade de maiores esforços de investigação interdisciplinar que propiciem o conhecimento integrado das relações entre sistemas humanos e naturais. Isso porque o enfrentamento das crises socioeconômicas e ambientais do século XXI - mudanças climáticas, perda de biodiversidade, pobreza persistente, etc. - exige abordagens inovadoras e que se distanciam do paradigma da ciência clássica newtoniana. Como avançar, então, no diálogo interdisciplinar e na formação de uma agenda de pesquisa efetivamente plural compatível com a natureza complexa dos fenômenos reais?

A partir deste problema de pesquisa, este artigo objetivou oferecer reflexões iniciais de como facilitar a realização de pesquisas interdisciplinares com foco na compreensão das interfaces entre sistemas naturais e socioeconômicos. Partindo-se da premissa básica de que os serviços ecossistêmicos são a interface básica entre capital natural e prosperidade humana, argumentou-se que a abordagem de serviços ecossistêmicos e a modelagem econômicoecológica (ASE e MEE, respectivamente) podem ser ferramentas úteis para este tipo de análise.

Do ponto de vista analítico e conceitual, a ASE destaca o papel dos ecossistemas e sua biodiversidade enquanto contribuintes originais para a geração do bem-estar humano. Do lado metodológico, a MEE apresenta-se como instrumento concreto para a operacionalização do conhecimento gerado por uma abordagem sistêmica e integradora das interações entre sociedade e natureza. Em conjunto, ambas as abordagens têm o potencial de direcionar o foco das análises que visam à compreensão das interfaces entre natureza e meio ambiente. Para além disso, a consideração conjunta da ASE e MME é coerente com os princípios da economia ecológica, a qual em última instância pode ser considerada a ciência que cuida da gestão da sustentabilidade e das oportunidades para as gerações futuras.

Esforços interdisciplinares de pesquisa não devem focar exclusivamente a geração de conhecimento teórico. Ao contrário, o acúmulo de conhecimento deve se desdobrar em aplicações práticas de políticas públicas cujo objetivo último seja a obtenção do chamado desenvolvimento sustentável. Com base neste argumento, este trabalho procurou apresentar em que medida o processo de elaboração de políticas pode se beneficiar de uma abordagem holística baseada no conceito de serviços ecossistêmicos e na modelagem econômico-ecológica.

\section{REFERÊNCIAS BIBLIOGRÁFICAS}

1. ANDRADE, D.C., ROMEIRO, A. R., SIMÕES, M.S. From an Empty to a Full World: a nova natureza da escassez e suas implicações. Economia e Sociedade, v. 21, p. 695-722, Campinas, 2012.

2. ANDRADE, D.C, ROMEIRO, A.R. Serviços ecossistêmicos e sua importância para o sistema econômico e o bem-estar humano. Instituto de Economia - Universidade Estadual de Campinas (UNICAMP), SP: Texto para Discussão 155. 2009.

3. BRAAT, L.C., DE GROOT, R. The ecosystem services agenda: bridging the worlds of natural science and economics, conservation and development, and public and private policy. Ecosystem Services, v. 1, p. 4-15, 2012.

4. CHECHIN, A.D., VEIGA, J.E. da. A economia ecológica e evolucionária de Georgescu-Roegen. 
Revista de Economia Política, São Paulo, v. 30, n. 3 (119), p. 438-454, julho-setemb2010.

5. COSTANZA, R. Changing the way we view humanity and the rest of nature. Solutions, v. 2, n. 6, p. 1, novembro, 2011.

6. COSTANZA, R., RUTH, M. Using dynamic modeling to scope environmental problems and build consensus. Environmental Management, v. 22, n. 2, p. 183-195, 1998.

7. COSTANZA, R., D'ARGE, R., DE GROOT, R.S., FARBER, S., GRASSO, M., HANNON, B., LIMBURG, K., NAEEM, S., O'NEILL, R.V., PARUELO, J., RASKIN, R.G., SUTTON, P., VAN DEN BELT, M. The value of the world's ecosystem services and natural capital. Nature, v. 387, p. 253-260, 1997.

8. DAILY, G. Nature's services: societal dependence on natural ecosystem. Washington D.C.: Island Press, 1997.

9. DE GROOT, R.S., WILSON, M.A., BOUMANS, R.M.J. A typology for the classification, description, and valuation of ecosystem functions, goods and services. Ecological Economics, v. 41, p. 393-408, 2002.

10. EFTEC (Economic for the Environment Consultancy). Economic, social and ecological value of ecosystem services: a literature review. Final report prepared for The Department for Environment, Food and Rural Affairs (Defra), 2005.

11. GEORGESCU-ROEGEN, N. The entropy law and the economic process. Cambridge: Harvard University Press, 1971.

12. GOMES, A.G., VARRIALE, M.C. Modelagem de ecossistemas: uma introdução. Santa Maria: Editora UFSM, 2004.

13. HARDIN, G. The tragedy of the commons. Science, v. 162, p. 1243-1248, 1968.

14. HEIN, L., VAN KOPPEN, K., DE GROOT, R.S., VAN IERLAND, E.C. Spatial scales, stakeholders and the valuation of ecosystem services. Ecological Economics, v. 57, n. 2, p. 209-228, 2006.

15. LANT, C.L., RUHL, J.B., KRAFT, S.E. The tragedy of ecosystem services. BioScience, v. 58, p. 969-974, 2008.

16. LEVIN, S. A. Ecosystems and the biosphere as complex adaptive systems. Ecosystems, v. 1, p. 431-436, 1998.

17. LIMBURG, K.E., FOLKE, C. The ecology of ecosystem services: introduction to the special issue. Ecological Economics, v. 29, p. 179-182, 1999.

18. MARTÍN-LÓPEZ, B. GÓMEZ-BAGGETHUN, E., LOMAS, P.L., MONTES, C. Effects of spatial and temporal scales on cultural services valuation. Journal of Environmental Management, v. 90, p. 1050-1059, 2009.

19. MEA (Millennium Ecosystem Assessment). Ecosystem and Human Well-Being: synthesis. Washington, D.C.: Island Press, 2005.

20. MEA (Millennium Ecosystem Assessment). Ecosystem and Human Well-Being: a framework for assessment. Washington, D.C.: Island Press, 2003.

21. MÜLLER, F., BURKHARD, B. The indicator side of ecosystem services. Ecosystem Services, v. 1, p. 26-30, 2012.

22. NAEEM, S., CHAPIN III, F.S., COSTANZA, R., EHRLICH, P.R., GOLLEY, F.B., HOOPER, D.U., LAWTON, J.H., O’NEILL, R.V., MOONEY, H.A., SALA, O.E., SYMSTAD, A.J., TILMAN, D. 
Biodiversity and ecosystem functioning: maintaining natural life support processes. Issues in Ecology, n. 4. Washington, D.C.: Ecological Society of America, 1999.

23. ÖZKAYNAK, B., ADAMAN, F., DEVINE, P. The identity of ecological economics: retrospect and prospects. Cambridge Journal of Economics, v. 36, p. 1123-1142, 2012.

24. ROMEIRO, A.R. Sustainable development and institutional change: the role of altruistic behavior. Texto para Discussão. Instituto de Economia/UNICAMP, n 97, junho, 2000.

25. RESENDE, F.M., FERNANDES, G.W., ANDRADE, D.C. A perigosa deterioração dos serviços de ecossistemas. Scientific American Brasil, v. 140, p. 70-75, janeiro, 2014.

26. SUKHDEV, P. The Economics of Ecosystems and Biodiversity. Interim Report of the Convention on Biological Diversity. Cambridge, United Kingdom: European Communities, 2008.

27. TANSLEY, A.G. The use and abuse of vegetational concepts and terms. Ecology, v. 3, p. 284307, 1935.

28. UN MILLENNIUM PROJECT. Investing in development: a practical plan to achieve the Millennium Development Goals. Overview. New York City: United Nations, 2005.

29. UNEP (United Nations Environment Programme). The convention on biological diversity: from conception to implementation. CBD News Special Edition. Secretariat of the Convention of Biological Diversity, 2004.

30. VOINOV, A. Systems science and modeling for ecological economics. Londres: Elsevier Inc, 2008.

31. VON BERTALANFFY, L. Teoria Geral dos Sistemas. Rio de Janeiro: Ed. Vozes, 1975.

32. WALLACE, K.J. Classification of ecosystem services: problems and solutions. Biological Conservation, v. 139, p. 235-246, 2007.

33. YORK, R., ROSA, E.A., DIETZ, T. Footprints on the Earth: the environmental consequences of modernity. American Sociological Review, v. 68, p. 279-300, 2003. 